\title{
A Mechanical Analogue of the Refracting Telescope
}

Maurizio Vannoni, Giuseppe Molesini, and Andrea Sordini, Istituto Nazionale di Ottica, Firenze, Italy

Samuele Straulino, University of Florence, 50019 Sesto Fiorentino, Italy

$\mathrm{T}$ he recent celebration of the discoveries made by Galileo four centuries ago has attracted new attention to the refracting telescope and to its use as an instrument for the observation of the night sky. ${ }^{1}$ This has offered the opportunity for addressing in the classroom the basic principles explaining the operation of the telescope. When doing so, a key concept that is faced is magnification. In geometrical optics, the treatment of magnification is generally given in terms of light rays and first-order (Gaussian or paraxial) ray tracing. Computer programs are available with which the light path through the lenses and the whole telescope can be simulated.

An alternative approach that also proves useful to explain magnification is by using a mechanical analogue that, without sacrificing the rigor of the treatment, is suitable to enhance understanding of the telescope's operation. Such an approach illustrates the mechanism of angular magnification by simple two-lever systems, easy to fabricate and useful to operate directly with students. In addition, relating optics concepts to mechanical devices, the approach is also useful to point out aspects that are common to different disciplines.

Here we briefly review the basic optics principles, and present the concept and the implementation of a mechanical analogue of the refracting telescope. The treatment is at the level of introductory physics college students, but can also be presented to younger classes and to the general public by appropriate tailoring of the more technical issues.

\section{Optics background}

The size we attribute to an object depends on the angle the object subtends at the eye: it is this angle that determines the extent of the image which is cast on the retina (Fig. 1). Magnification with telescopes is achieved by increasing this angle. This was clear from the very beginning, as Galileo wrote in The Assayer: "The telescope shows the objects larger because it brings

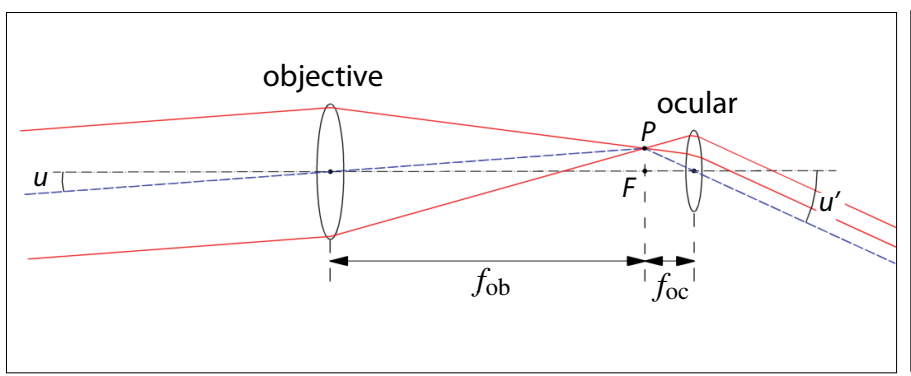

Fig. 2. Schematic layout of the Keplerian telescope showing the geometrical construction of the angles $u, u^{\prime}$ by means of the principal rays (blue dashed lines).

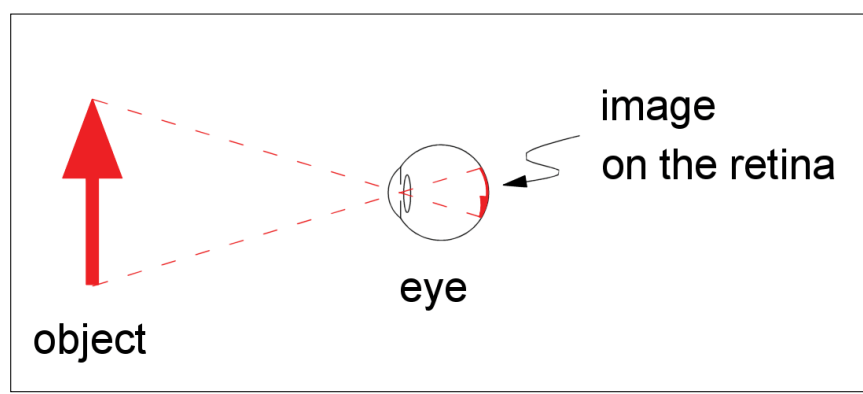

Fig. 1. Apparent size of an object seen by the eye. The image cast on the retina is larger if the object subtends a larger angle.

them under a larger angle than when they are seen without the instrument." ${ }^{2}$

As to the detailed mechanism by which such an angular magnification is achieved, one may initially refer to a Keplerian (astronomical) telescope. While such a telescope was devised and made at a later time than the Galilean one, its working principle is easier to explain. Following classical treatments, here we make use of the geometrical layout based on principal rays. ${ }^{3-5}$ Referring to Fig. $2, F$ is the focal point common to the objective and the ocular. Light coming from a far distance at an angle $u$ to the axis is focused at a point $P$ above $F ; P$ is determined by tracing the principal ray (blue dashed line) that passes through the objective center and then proceeds undeviated to the common focal plane where $F$ lies. It is here noticed that principal rays are not necessarily intended as effective light paths but are usually drawn to geometrically construct the image (location, extent) in paraxial optics. A similar construction is used to find the direction of the (parallel) rays exiting the ocular. The angle $u^{\prime}$ between these rays and the optical axis is formed by tracing the new principal ray from $P$ through the ocular center, undeviated by the lens. The magnification $m$ of the telescope is

$$
m=u^{\prime} / u \cong f_{\mathrm{ob}} / f_{\mathrm{oc}} \text {, }
$$




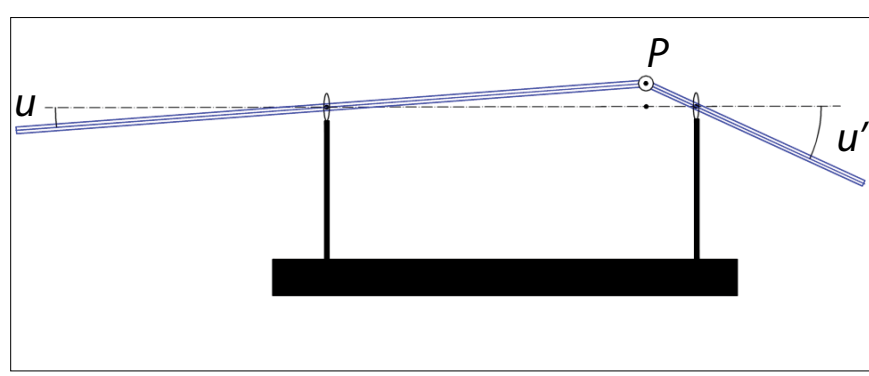

Fig. 4. Mechanical analogue of the Keplerian telescope.

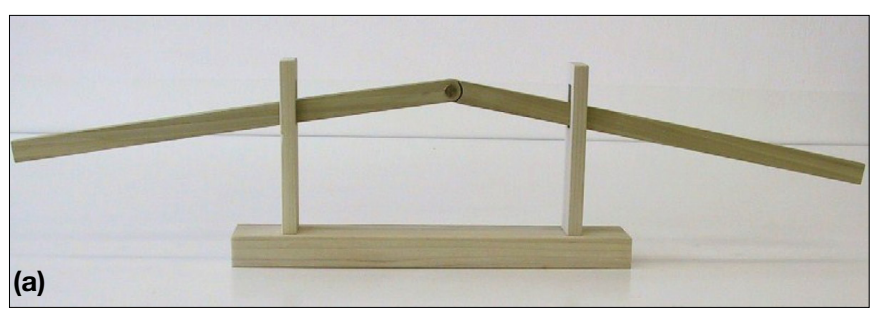
Fig. 6. Photographs of the mechanical analogues used for d
where $f_{\mathrm{ob}}$ is the focal length of the objective and $f_{\mathrm{oc}}$ that
of the ocular. An actual increase of the image size is then achieved for $u^{\prime}>u$, i.e., for $f_{\mathrm{ob}}>f_{\mathrm{oc}}$. As a feature of the Keplerian telescope, it is also noted that if the angle $u$ is clockwise with respect to the optical axis, the angle $u^{\prime}$ is counterclockwise; since such angles are discordant, the observed image is inverted.

The interplay between the angles $u, u^{\prime}$ determining the angular magnification of the telescope is immediately understood if we refer to the two principal rays only. Such principal rays, separately drawn for the objective and the ocular, serve as direction guidelines for the light beam prior to entering the telescope and after exiting.

Similar drawings and considerations can be made in the case of a Galilean telescope (Fig. 3), with the difference that the intermediate focal point $P$ is virtual, and the angles $u, u^{\prime}$ are concordant, so that the observed image is erect.

\section{Mechanical analogues}

The magnifying mechanism can be described in terms of the movement of two levers with fulcrums at the centers of the lenses and hinged together at the common point $P$; such levers represent the principal rays discussed above. Figure 4 shows the case of the Keplerian telescope and Fig. 5 the case of the Galilean one. The left lever is associated to the objective and the right lever to the ocular. The analogues of the focal lengths $f_{\mathrm{ob}}$ and $f_{\mathrm{oc}}$ are the lengths of the levers from $P$ to their fulcrums. Moving the hinge up and down, the working mechanism of the levers provides the same angular magnification as the objective-ocular pair of a telescope $\left(m \cong f_{\mathrm{ob}} / f_{\mathrm{oc}}\right)$.

The models in the figures above have been made for dem-

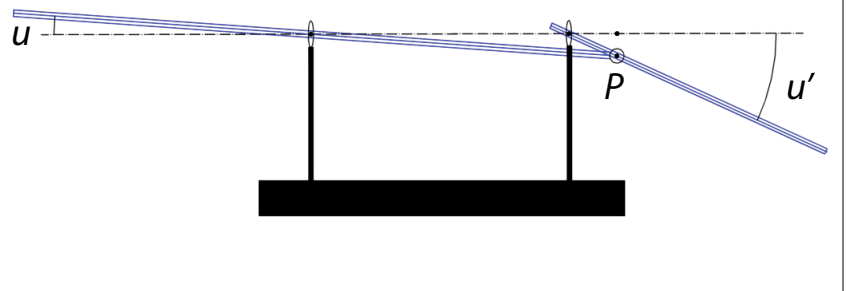

Fig. 5. Mechanical analogue of the Galilean telescope.

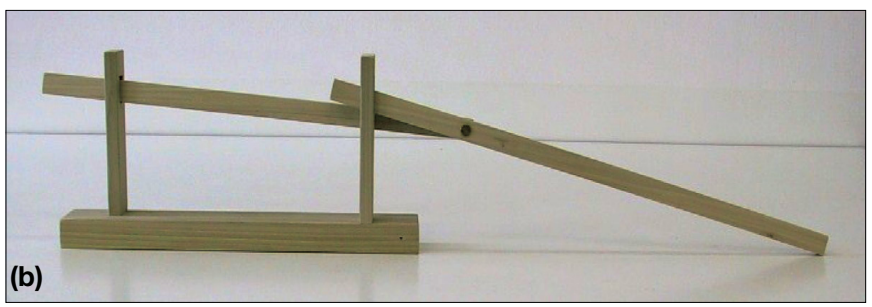

(b) onstration using wooden slats for the levers and supports, and simple metal parts for the pivots (Fig. 6). Again, the slats do not indicate actual optical paths, but represent the direction of the incoming and exiting rays.

The angular magnification can be changed by making the slats to slide in their retaining holes, and then again moving the hinge up and down for operation. It can easily be shown that the magnifying effect increases when the pivot is moved closer to the ocular fulcrum. Using such mechanical models and making students play with them results in a valid aid to effectively visualize the basic features of the refracting telescopes, and to help them understand even in the absence of a specific background in optics.

\section{Acknowledgments}

The authors are grateful to Romano Straulino and Pietro Zito for ingenious work with the construction of the wooden models used for demonstration.

\section{References}

1. S. M. Pompea and D. Isbell, "The International Year of Astronomy 2009: New approaches and novel resources for physics classrooms," Phys. Teach. 47, 428-433 (Oct. 2009).

1. Galileo Galilei, Il Saggiatore (1623), Chap. XII. Translation by the authors.

2. F. Miller Jr., College Physics, 5th ed. (Harcourt Brace Jovanovich, New York, 1982), pp. 580-583.

3. J. D. Wilson and J. Kinard, College Physics (Allyn and Bacon, Boston, 1990), pp. 631-633.

4. S. Allen, "The Gaussian formula and the elusive fourth principal ray," Am. J. Phys. 60, 160-163 (Feb. 1992). 